\title{
Besouros de solo (Insecta: Coleoptera) em fragmento de mata de restinga no extremo sul do Brasil
}

\author{
Leandro Encarnação Garcia ${ }^{1 *}$ \\ Rodrigo Milton Moraes ${ }^{2}$ \\ Élvia Elena Silveira Vianna ${ }^{1}$ \\ ${ }^{1}$ Programa de Pós-Graduação em Entomologia, Universidade Federal de Pelotas \\ Campus Capão do Leão, s/n, CEP 96160-000, Capão do Leão - RS, Brasil \\ ${ }^{2}$ Programa de Pós-Graduação em Biologia Animal, Universidade Federal do Rio Grande do Sul \\ Avenida Paulo Gama, 110, Farroupilhas, CEP 90040-060, Porto Alegre - RS, Brasil \\ * Autor para correspondência \\ leandrogarcia20504@gmail.com
}

Submetido em 24/05/2016

Aceito para publicação em 09/11/2016

\section{Resumo}

Coleoptera é um dos grupos taxonômicos mais diversos com cerca de 390.000 espécies descritas. Devido à grande diversidade e concentração de especialistas em poucos grupos, torna-se difícil realizar o inventário da fauna de Coleoptera. Este trabalho visa contribuir com o conhecimento da diversidade de besouros em matas de restinga, localizadas no extremo sul do Rio Grande do Sul, Brasil. O objetivo deste estudo foi inventariar as espécies de coleópteros de solo amostradas com armadilhas de queda, durante o período de janeiro de 2011 a janeiro de 2012, bem como avaliar a abundância, sazonalidade e riqueza do grupo. Foi amostrado um total de 6.954 coleópteros distribuídos em 25 famílias e 89 morfoespécies. Dos indivíduos amostrados, 63,79\% pertenceram à família Nitidulidae; $14,83 \%$ à Ptiliidae e; 6,82\% à Staphylinidae. Dezenove famílias tiveram menos de $1 \%$ de indivíduos amostrados. Em relação ao número de morfoespécies por família 29,21\% pertenceram à Curculionidae; $14,61 \%$ à Carabidae; $10,11 \%$ a Chrysomelidae; 6,74\% à Nitidulidae e Scarabaeidae, ambas com mesmo percentual. Neste trabalho, foram verificadas duas situações sazonais onde a primavera teve o mesmo número de espécies que o verão e maior número de indivíduos amostrados.

Palavras-chave: Armadilha de solo; Diversidade; Estações; Levantamento; Sul do Brasil

\section{Abstract}

Ground beetles (Insecta: Coleoptera) at a restinga forest fragment in far-southern Brazil. Coleoptera is one of the most varied taxonomic groups, it has about 390,000 described species. Due to the wide variety and concentration of experts in a few groups, it becomes hard to carry out the inventory of Coleoptera fauna. This paper aims to contribute to knowledge on the diversity of beetles in restinga forests located in far-southern Brazil. The goal of this study was surveying the species of ground beetles sampled with pitfall traps, within the period from January 2011 to January 2012, as well as evaluating the group's abundance, seasonality, and richness. A total of 6,954 ground beetles distributed into 25 families and 89 morphospecies were sampled. Out of these individuals, $63.79 \%$ belong to the Nitidulidae family; $14.83 \%$ to the Ptiliidae; and $6.82 \%$ to the Staphylinidae. Nineteen families had less than $1 \%$ of sampled individuals. Regarding the number of morphospecies per family, 
$29.21 \%$ belong to the Curculionidae; $14.61 \%$ to the Carabidae; $10.11 \%$ to the Chrysomelidae; $6.74 \%$ to the Nitidulidae; and $6.74 \%$ to the Scarabaeidae. In this research, two seasonal situations were observed, where the spring had the same number of species than summer and a larger number of sampled individuals.

Key words: Diversity; Pitfall trap; Seasons; Southern Brazil; Survey

\section{Introdução}

Coleoptera, descrita por Linnaeus em 1758, é um dos grupos taxonômicos mais diversos de organismos, com 387.100 espécies (SLIPINSKI et al., 2011). No Brasil, são registradas cerca de 30.000 espécies de besouros (LEWINSOHN; PRADO, 2005) em 105 famílias (CASARI; IDE, 2012). Pela sua diversidade e abundância, os besouros representam grande parte da biomassa de um ecossistema, sendo um importante recurso alimentar para outros invertebrados e para diversos grupos de vertebrados como aves, lagartos e pequenos roedores (SPEIGHT et al., 1999). São utilizados direta e indiretamente na conservação de hábitats e utilizados em controle biológico. Além dos hábitos alimentares mais variados possíveis, encontram-se os decompositores de matéria orgânica vegetal e animal, fitofagia, predadores de sementes e de outros invertebrados (KEVAN; BAKER, 1983; SPEIGHT et al., 1999; SCHERER; ROMANOWSKI, 2005; NOGUEIRA; ARRUDA, 2006; TRIPLEHORN; JOHNSON, 2011).

Some-se, ainda, a merecida atenção que esses insetos despertam para as pesquisas, pela facilidade de amostragem, pela ampla distribuição, por ocuparem diversos extratos vegetais, pela facilidade de reconhecimento, pela abundância em vários sistemas ecológicos, resposta rápida às modificações ambientais e/ou distúrbios antrópicos (PEARSON, 1994). Este trabalho visa contribuir com o conhecimento da biodiversidade de besouros localizados no extremo sul do Rio Grande do Sul, Brasil. Portanto, o objetivo foi inventariar as espécies de coleópteros, coletados com armadilhas de solo em um fragmento de restinga, bem como avaliar a abundância, sazonalidade e riqueza do grupo.

\section{Material e Métodos}

\section{Área de estudo}

O estudo foi realizado em fragmento de 23 ha de Mata de Restinga, situada a $3 \mathrm{~km}$ do Campus da Universidade Federal de Pelotas - UFPel (31 ${ }^{\circ} 48^{\prime} 58^{\prime \prime} \mathrm{S}$ e $52^{\circ} 25^{\prime} 55^{\prime}$ 'W), município de Capão do Leão, planície costeira (extremo-sul), RS, Brasil (Figura 1). Essa área é uma unidade de preservação federal permanente de responsabilidade da Universidade Federal de Pelotas,

FIGURA 1: Área amostral de restinga para captura de coleópteros no período de janeiro de 2011 a janeiro de 2012, no extremo sul do Rio Grande do Sul, Brasil. Os pontos azuis são os locais das armadilhas instaladas.

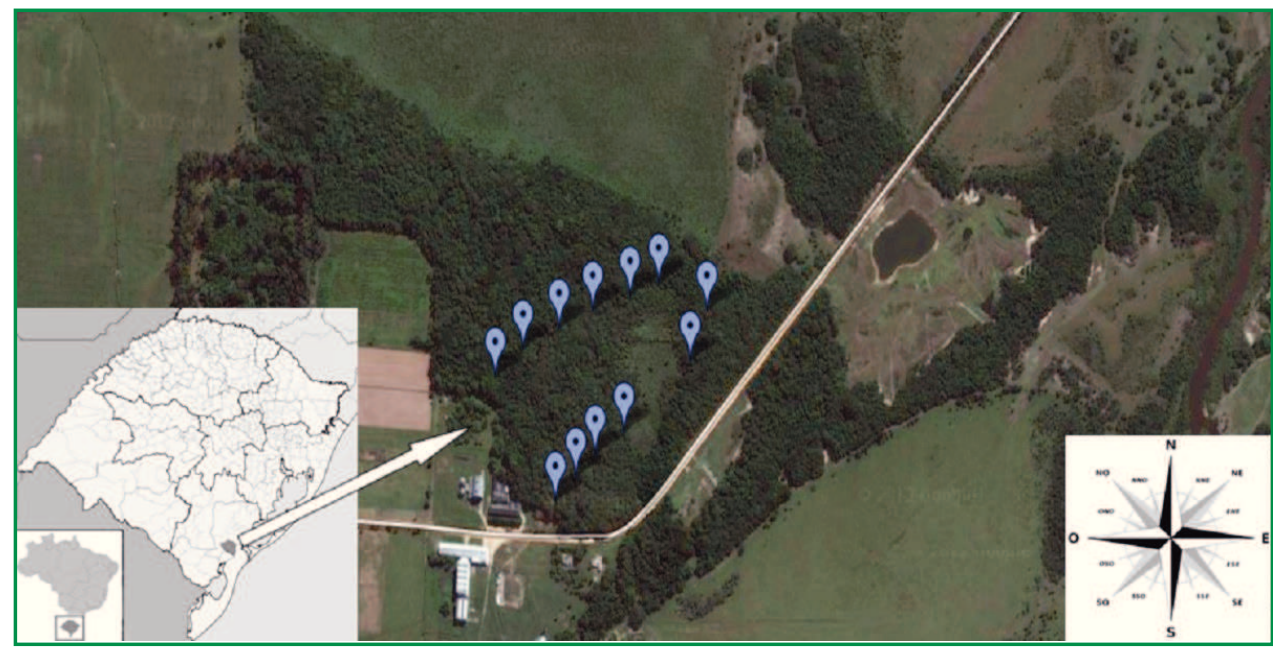


regulamentada pela Portaria Ministerial 77, no ano de 1964.

A área de estudo faz parte do bioma Pampa e se encontra na fisionomia das Formações Pioneiras com forte influência da Floresta Estacional Semidecidual (VELOSO et al., 1991). O clima é mesotérmico, sempre úmido, com verões quentes, de acordo com a classificação de Köppen-Geiger (PEEL et al., 2007). $\mathrm{Na}$ área de estudo, ocorre a presença de uma espécie vegetal exótica Asparagus setaceus (Kunth) Jessop, apresentando histórico de perturbação antrópica, tendo passado por um processo paisagístico na metade do século XX (LUIS; BERTELS, 1951).

\section{Coleta e processamento de dados}

Para a distribuição das armadilhas foram traçados dois transectos de amostragem, distanciados entre si $120 \mathrm{~m}$ (da borda para o exterior). Em cada transecto foram instaladas seis armadilhas de queda, com distância de 50 $m$ entre elas, totalizando 12 armadilhas. Foram utilizados frascos de vidro com capacidade de $350 \mathrm{~mL}$ e de $5,5 \mathrm{~cm}$ de diâmetro contendo solução de $100 \mathrm{~mL}$ de formaldeído a 5\%. Não foram utilizados atrativos. As armadilhas foram instaladas na primeira quinzena de janeiro de 2011, onde eram retiradas a cada três semanas e realizada reposição da solução de formaldeído, perdurando 1 ano de amostragem. No total foram realizadas 17 coletas, divididas pelas estações do ano: verão (4), outono (5), inverno (4) e primavera (4). Após a coleta, o material foi triado, quantificado, identificado e morfotipado com utilização de microscópio estereoscópico, chave de identificação (TRIPLEHORN; JOHNSON, 2011) e consulta a especialistas. Os insetos foram depositados na coleção do laboratório de Entomologia, UFPel.

A eficiência da amostragem foi avaliada através da curva de acumulação de espécies e seu intervalo de confiança, em que foram relacionadas as amostras com a riqueza observada. Foram utilizados os estimadores de riqueza Chao 2, Jackknife 1 e Jackknife 2 para verificar a representatividade das assembleias de besouros na área de estudo. Esses estimadores são baseados na incidência de espécies por amostras, sendo os mais confiáveis para poucas amostras. A dominância foi estimada segundo o índice de Simpson (1-D), e a diversidade, de acordo com o índice de Shannon-Wiener $(\mathrm{H})$.

Foi realizada análise de similaridade (Oneway ANOSIM), que é um teste não paramétrico, aqui utilizado para verificar a diferença na riqueza e abundância de besouros. Foram utilizados coeficientes de similaridade, para composição de espécies (índice de Simpson), e de abundância (índice de Bray-Curtis) entre os quatro grupos de dados coletados (primavera, verão, outono e inverno). $\mathrm{O}$ valor do teste " $\mathrm{R}$ " varia de 0 a 1 , em que o valor de $\mathrm{R}$ próximo de 1 é dissimilar (grupos diferentes) e o valor próximo de 0 é similar (grupos não exibem diferença) (CLARKE, 1993). Também foram realizadas permutações das similaridades esperadas (9.999 réplicas), comparando com o valor de $\mathrm{R}$ obtido para cada grupo, com uma significância $\alpha=0,05 \%$, gerando um p-valor para cada comparação entre os grupos. Todas as análises foram realizadas através do programa Past versão 2.17 (HAMMER et al., 2001). Foram obtidas as médias das temperaturas máximas e mínimas das estações do ano durante o período de coleta (EMBRAPA, 2016).

\section{Resultados}

Ao longo das estações, foi amostrado um total de 6.954 coleópteros distribuídos em 25 famílias (Tabela 1) e em 89 morfoespécies (Tabela 2). Dos indivíduos amostrados, $4.436(63,79 \%)$ pertenceram à família Nitidulidae; 1.031 (14,83\%), à Ptiliidae; 474 (6,82\%), à Staphylinidae; 269 (3,87\%), à Carabidae; 248 (3,57\%), à Scydmaenidae e 206 indivíduos (2,96\%), à Curculionidae. Das 25 famílias, 19 tiveram menos de $1 \%$ de indivíduos amostrados. Em relação à riqueza de espécies, as três famílias mais diversas na amostragem foram Curculionidae, com 26 morfoespécies $(29,21 \%)$, seguida de Carabidae (14,61\%) e Chrysomelidae $(10,11 \%)$. 
TABELA 1: Relação do número de indivíduos, espécies e percentual de coleópteros amostrados no período de janeiro de 2011 a janeiro de 2012, com armadilhas de queda, em área de restinga, no extremo sul do Rio Grande do Sul, Brasil (N, número de indivíduos; \%, porcentagem em relação total; $\mathrm{S}$, número de espécies).

\begin{tabular}{lcccc}
\hline \multicolumn{1}{c}{ Família } & $\mathbf{N}$ & $\mathbf{\%}$ & $\mathbf{S}$ & $\mathbf{\%}$ \\
\hline Anobiidae & 1 & 0,01 & 1 & 1,12 \\
Carabidae & 269 & 3,87 & 13 & 14,61 \\
Cerambycidae & 1 & 0,01 & 1 & 1,12 \\
Chrysomelidae & 34 & 0,49 & 9 & 10,11 \\
Corylophidae & 47 & 0,68 & 1 & 1,12 \\
Curculionidae & 206 & 2,96 & 26 & 29,21 \\
Dryopidae & 2 & 0,03 & 1 & 1,12 \\
Dytiscidae & 3 & 0,04 & 2 & 2,25 \\
Elateridae & 29 & 0,42 & 5 & 5,62 \\
Hybosoridae & 57 & 0,82 & 1 & 1,12 \\
Hydrophilidae & 1 & 0,01 & 1 & 1,12 \\
Lampyridae & 1 & 0,01 & 1 & 1,12 \\
Latriidae & 2 & 0,03 & 1 & 1,12 \\
Leiodidae & 49 & 0,7 & 1 & 1,12 \\
Lucanidae & 16 & 0,23 & 1 & 1,12 \\
Melolonthidae & 1 & 0,01 & 1 & 1,12 \\
Mordellidae & 1 & 0,01 & 1 & 6,74 \\
Nitidulidae & 4436 & 63,79 & 6 & 3,37 \\
Ptiliidae & 1031 & 14,83 & 3 & 6,74 \\
Scarabaeidae & 12 & 0,17 & 6 & 3,37 \\
Scirtidae & 7 & 0,1 & 3 & 3,37 \\
Scydmaenidae & 248 & 3,57 & 3 & - \\
Staphylinidae & 474 & 6,82 & -12 \\
Tenebrionidae & 0,3 & $\mathbf{1 0 0}$ \\
Trogidae & 21 & 0,07 & $\mathbf{8 9}$ & \\
\hline Total & 5954 & $\mathbf{1 0 0}$ & & \\
\hline
\end{tabular}

Foi possível observar que a curva de acúmulo de espécies possui um crescimento contínuo, mas ainda não chega a estabilizar (Figura 2). Com relação aos estimadores de riqueza, apenas Jackknife 2 na estação verão e Chao 2 na primavera possuem valores menores que $80 \%$ das espécies amostradas. Do total de coleópteros, $49,7 \%$ foram amostrados na primavera e $30,3 \%$ no verão, somando $80 \%$ dos indivíduos amostrados nessas estações. 
TABELA 2: Lista de morfoespécies de coleópteros amostradas nas diferentes estações com armadilha de queda, entre janeiro de 2011 e janeiro de 2012, em fragmento de restinga, no extremo sul do Rio Grande do Sul, Brasil.

\begin{tabular}{|c|c|c|c|c|c|c|}
\hline Família & Morfoespécie & Verão & Outono & Inverno & Primavera & Total \\
\hline Anobiidae & Anobiidae sp. & 0 & 0 & 0 & 1 & 1 \\
\hline \multirow[t]{13}{*}{ Carabidae } & Cnemalobus striatus & 5 & 5 & 0 & 6 & 16 \\
\hline & Colliuris sp.1 & 0 & 0 & 1 & 0 & 1 \\
\hline & Cynthidia sp. & 9 & 2 & 34 & 66 & 111 \\
\hline & Galerita melanarthra & 0 & 1 & 0 & 0 & 1 \\
\hline & Lebia $\mathrm{sp} .1$ & 3 & 0 & 0 & 0 & 3 \\
\hline & Lebia sp.2 & 4 & 0 & 7 & 80 & 91 \\
\hline & Lebia $\mathrm{sp} .3$ & 1 & 0 & 0 & 2 & 3 \\
\hline & Pseudabarys sp.1 & 5 & 0 & 2 & 7 & 14 \\
\hline & Pseudabarys sp. 2 & 0 & 0 & 1 & 5 & 14 \\
\hline & Pentagonica sp. 1 & 0 & 0 & 2 & 12 & 6 \\
\hline & Pterostichini sp. 1 & 0 & 0 & 0 & 2 & 2 \\
\hline & Pterostichini sp. 2 & 0 & 0 & 2 & 0 & 2 \\
\hline & Scarites sp.1 & 0 & 0 & 0 & 5 & 5 \\
\hline Cerambycidae & Lamiinae sp.1 & 1 & 0 & 0 & 0 & 1 \\
\hline \multirow[t]{9}{*}{ Chrysomelidae } & Chrysomelidae sp.1 & 3 & 0 & 3 & 20 & 26 \\
\hline & Chrysomelidae sp.2 & 0 & 0 & 1 & 0 & 1 \\
\hline & Chrysomelidae sp.3 & 0 & 0 & 0 & 1 & 1 \\
\hline & Chrysomelidae sp.4 & 0 & 0 & 0 & 1 & 1 \\
\hline & Chrysomelidae sp.5 & 1 & 0 & 0 & 0 & 1 \\
\hline & Chrysomelidae sp.6 & 0 & 0 & 0 & 1 & 1 \\
\hline & Colaspis sp.1 & 1 & 0 & 0 & 0 & 1 \\
\hline & Galerucinae sp. 1 & 0 & 0 & 0 & 1 & 1 \\
\hline & Galerucinae sp.2 & 1 & 0 & 0 & 0 & 1 \\
\hline Corylophidae & Corylophidae sp.1 & 10 & 4 & 7 & 26 & 47 \\
\hline \multirow[t]{22}{*}{ Curculionidae } & Curculionidae sp.1 & 2 & 0 & 0 & 0 & 2 \\
\hline & Curculionidae sp.2 & 14 & 29 & 0 & 4 & 47 \\
\hline & Curculionidae sp.3 & 1 & 0 & 0 & 0 & 1 \\
\hline & Curculionidae sp.4 & 2 & 0 & 2 & 4 & 8 \\
\hline & Curculionidae sp.5 & 2 & 0 & 0 & 0 & 2 \\
\hline & Curculionidae sp.6 & 1 & 0 & 0 & 0 & 1 \\
\hline & Curculionidae sp.7 & 1 & 1 & 0 & 0 & 2 \\
\hline & Curculionidae sp. 8 & 9 & 33 & 13 & 24 & 79 \\
\hline & Curculionidae sp.9 & 1 & 0 & 2 & 0 & 3 \\
\hline & Curculionidae sp.10 & 1 & 0 & 0 & 0 & 1 \\
\hline & Curculionidae sp.11 & 6 & 1 & 0 & 5 & 12 \\
\hline & Curculionidae sp.12 & 2 & 1 & 6 & 5 & 14 \\
\hline & Curculionidae sp.13 & 1 & 3 & 1 & 0 & 5 \\
\hline & Curculionidae sp.14 & 1 & 4 & 0 & 1 & 6 \\
\hline & Curculionidae sp.15 & 3 & 0 & 0 & 2 & 5 \\
\hline & Curculionidae sp.16 & 0 & 1 & 0 & 3 & 4 \\
\hline & Curculionidae sp.17 & 0 & 1 & 0 & 0 & 1 \\
\hline & Curculionidae sp.18 & 0 & 1 & 0 & 0 & 1 \\
\hline & Curculionidae sp.19 & 0 & 1 & 0 & 0 & 1 \\
\hline & Curculionidae sp.20 & 0 & 0 & 0 & 3 & 3 \\
\hline & Curculionidae sp.21 & 0 & 0 & 0 & 1 & 1 \\
\hline & Curculionidae sp.22 & 0 & 0 & 0 & 1 & 1 \\
\hline
\end{tabular}




\begin{tabular}{|c|c|c|c|c|c|c|}
\hline & Curculionidae sp.23 & 0 & 0 & 0 & 3 & 3 \\
\hline & Curculionidae sp.24 & 0 & 0 & 0 & 1 & 1 \\
\hline & Curculionidae sp.25 & 1 & 0 & 0 & 0 & 1 \\
\hline & Curculionidae sp.26 & 0 & 0 & 0 & 1 & 1 \\
\hline Dryopidae & Dryopidae sp.1 & 0 & 0 & 2 & 0 & 2 \\
\hline \multirow[t]{2}{*}{ Dytiscidae } & Dytiscidae sp.1 & 1 & 0 & 0 & 0 & 1 \\
\hline & Dytiscidae sp.2 & 1 & 1 & 0 & 0 & 2 \\
\hline \multirow[t]{5}{*}{ Elateridae } & Elateridae sp. 1 & 6 & 0 & 0 & 8 & 14 \\
\hline & Elateridae sp.2 & 3 & 0 & 0 & 3 & 6 \\
\hline & Elateridae sp.3 & 3 & 3 & 0 & 1 & 7 \\
\hline & Elateridae sp.4 & 1 & 0 & 0 & 0 & 1 \\
\hline & Elateridae sp.5 & 0 & 0 & 0 & 1 & 1 \\
\hline Hybosoridae & Hybosoridae sp.1 & 9 & 0 & 0 & 48 & 57 \\
\hline Hydrophilidae & Hydrophilidae sp.2 & 0 & 1 & 0 & 0 & 1 \\
\hline Lampiridae & Lampiridae sp.1 & 1 & 0 & 0 & 0 & 1 \\
\hline Latridiidae & Latridiidae sp.1 & 0 & 2 & 0 & 0 & 2 \\
\hline Leoididae & Dissochaetus sp.1 & 2 & 29 & 13 & 5 & 49 \\
\hline Lucanidae & Metadorcus rotundatus & 15 & 1 & 0 & 0 & 16 \\
\hline Melolonthidae & Melolonthidae sp.1 & 1 & 0 & 0 & 0 & 1 \\
\hline Mordellidae & Tomoxia sp.1 & 0 & 0 & 0 & 1 & 1 \\
\hline \multirow[t]{6}{*}{ Nitidulidae } & Stelidota geminata & 1443 & 360 & 316 & 2267 & 2 \\
\hline & Lobiopa insularis & 26 & 1 & 4 & 12 & 1 \\
\hline & Carpophilus sp.1 & 0 & 0 & 0 & 1 & 1 \\
\hline & Nitidulidae sp.1 & 0 & 0 & 0 & 3 & 4386 \\
\hline & Colopterus sp.1 & 1 & 0 & 0 & 0 & 43 \\
\hline & Carpophilidae sp.1 & 2 & 0 & 0 & 0 & 3 \\
\hline \multirow[t]{3}{*}{ Ptiliidae } & Ptiliidae sp.1 & 231 & 155 & 115 & 523 & 1024 \\
\hline & Ptiliidae sp.2 & 1 & 1 & 0 & 3 & 5 \\
\hline & Ptiliidae sp.3 & 0 & 1 & 0 & 1 & 2 \\
\hline \multirow[t]{6}{*}{ Scarabaeidae } & Dynastinae sp.1 & 0 & 2 & 0 & 0 & 3 \\
\hline & Ataenius sp.1 & 0 & 0 & 2 & 1 & 3 \\
\hline & Cyclocephala sp.1 & 1 & 0 & 0 & 0 & 1 \\
\hline & Dichotomius sp.1 & 1 & 1 & 0 & 0 & 1 \\
\hline & Canthon sp.1 & 0 & 0 & 0 & 3 & 2 \\
\hline & Scarabaeinae sp.1 & 0 & 0 & 0 & 1 & 2 \\
\hline \multirow[t]{3}{*}{ Scirtidae } & Scirtidae sp.1 & 2 & 0 & 2 & 1 & 1 \\
\hline & Scirtidae sp. 2 & 1 & 0 & 0 & 0 & 1 \\
\hline & Scirtidae sp.3 & 0 & 0 & 0 & 1 & 5 \\
\hline \multirow[t]{3}{*}{ Scydmaenidae } & Scydmaenidae sp.1 & 17 & 13 & 80 & 118 & 228 \\
\hline & Scydmaenidae sp.2 & 4 & 1 & 1 & 11 & 17 \\
\hline & Scydmaenidae sp.3 & 3 & 0 & 0 & 0 & 3 \\
\hline \multirow[t]{3}{*}{ Staphylinidae } & Staphylinidae spp. & 225 & 58 & 40 & 131 & 454 \\
\hline & Pselaphinae sp.1 & 3 & 0 & 0 & 13 & 16 \\
\hline & Pselaphinae sp.2 & 2 & 0 & 0 & 2 & 4 \\
\hline Tenebrionidae & Tenebrionidae spp. & 15 & 0 & 0 & 6 & 21 \\
\hline Trogidae & Polynoncus sp.1 & 0 & 0 & 5 & 0 & 5 \\
\hline Total & & 2113 & 718 & 664 & 3459 & 6954 \\
\hline
\end{tabular}


FIGURA 2: Curva de acúmulo de espécies de coleópteros amostradas no período de janeiro de 2011 a janeiro de 2012, com armadilhas de queda, em área de restinga, no extremo sul do Rio Grande do Sul, Brasil.

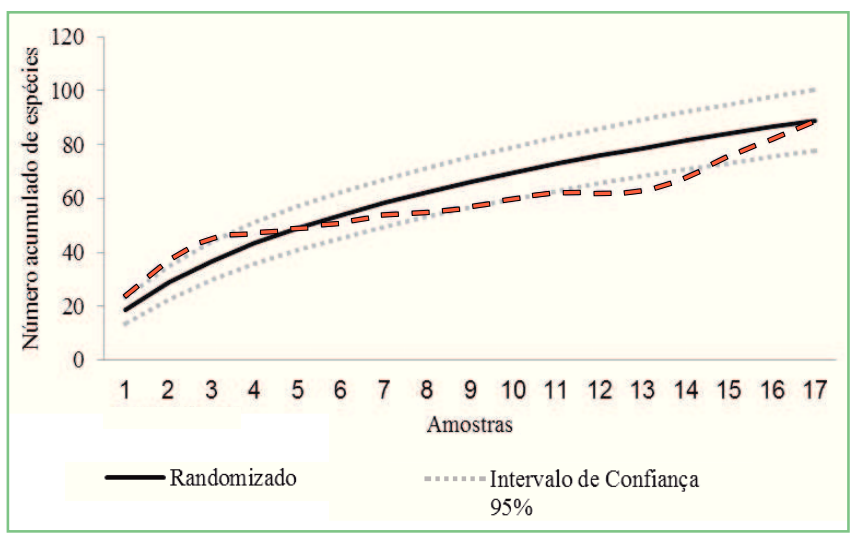

Foi comparada a abundância de espécimes entre as estações do ano de acordo com as zonas temperadas (estações do ano bem definidas), e a primavera foi a estação com o maior número de indivíduos (Figura 3). A análise de similaridade, verificando-se a abundância, dados do triângulo inferior da tabela, mostra que a abundância de indivíduos é menor com significância no outono (Tabela 4). Com relação à riqueza de espécies (Figura 4), a rarefação não evidencia diferença entre as coletas realizadas na primavera e no verão (Tabela 4), ambas com 52 espécies amostradas, apesar das temperaturas médias serem maiores no verão (Tabela 3).

FIGURA 3: Média de espécimes de coleópteros amostrados no período de janeiro de 2011 a janeiro de 2012, com armadilhas de queda, em área de restinga, no extremo sul do Rio Grande do Sul, Brasil.

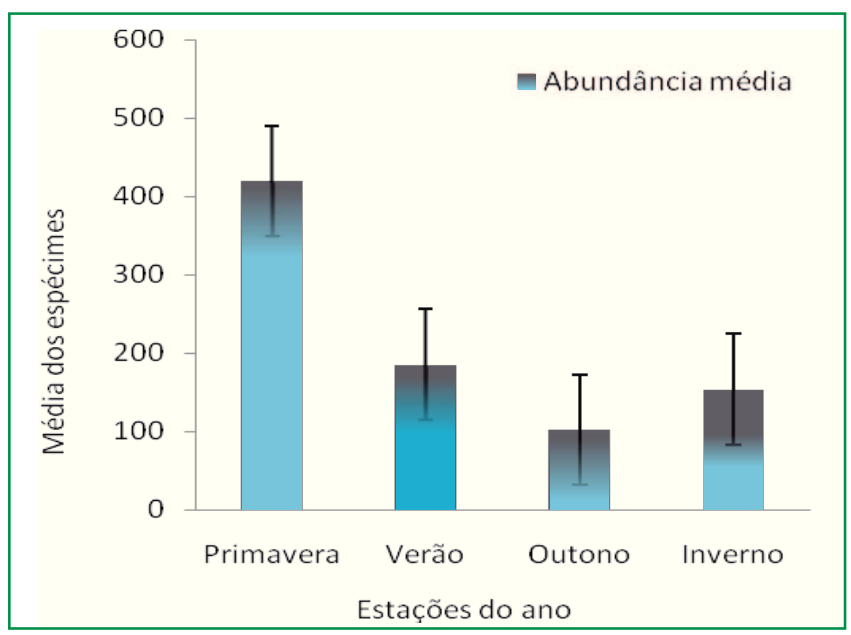

FIGURA 4: Rarefação das espécies de coleópteros amostradas no período de janeiro de 2011 a janeiro de 2012, com armadilhas de queda, em área de restinga, no extremo sul do Rio Grande do Sul, Brasil (Intervalo de Confiança $=95 \%$ ).

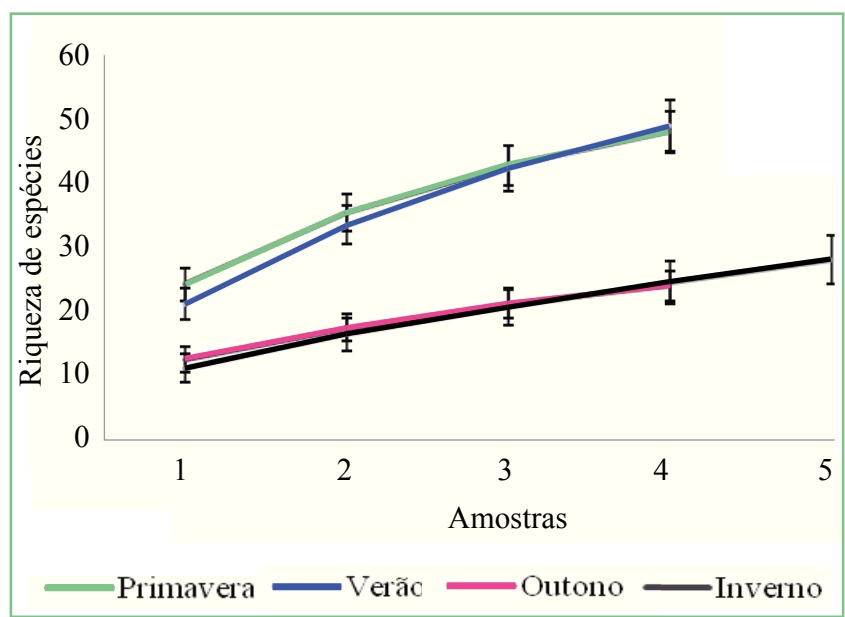

TABELA 3: Temperatura média, máximas e mínimas das estações do ano para as espécies de coleópteros amostradas nas diferentes estações com armadilha de queda, entre janeiro de 2011 e janeiro de 2012, em fragmento de Restinga, no extremo sul do Rio Grande do Sul, Brasil.

\begin{tabular}{lcccc}
\hline Temperatura & Verão & Outono & Inverno & Primavera \\
\hline Média ${ }^{\circ} \mathbf{C}$ & 22,8 & 15,9 & 12,3 & 18,7 \\
Mínima $^{\circ} \mathbf{C}$ & 18,1 & 12,5 & 7,4 & 15,0 \\
Máxima $^{\circ} \mathbf{C}$ & 26,0 & 20,0 & 18,2 & 22,5 \\
\hline
\end{tabular}

A análise de similaridade, utilizando os índices de Bray-Curtis com $\mathrm{R}=0,2097$ e índice de Simpson com $R=0,2077$, evidencia que tanto a riqueza como a abundância são similares entre as estações. Utilizando o teste de permutação dos valores de $\mathrm{R}$, para a riqueza de espécies, a similaridade verificou diferença estatística entre primavera e outono, com $p=0,022$, e entre inverno e outono, com $p=0,0241$ (Tabela 4, triângulo superior). As coletas realizadas no outono foram representadas por 30 espécies, e no inverno por 25 espécies.

O teste de permutação de ANOSIM (Tabela 4, triângulo inferior) confirmou a abundância média (Figura 3) dos coleópteros, indicando diferença estatística entre outono e verão, $\operatorname{com} p=0,0347$, e entre primavera e outono, $\operatorname{com} \mathrm{p}=0,0157$. 
TABELA 4: Análise de Similaridade (ANOSIM) para índices de Bray-Curtis (Triângulo inferior, valores abaixo do zero) e Simpson (Triângulo superior, valores acima do zero) entre as estações para fauna de coleópteros amostrada no período de janeiro de 2011 a janeiro de 2012, com armadilhas de queda, em área de restinga, no extremo sul do Rio Grande do Sul, Brasil.

\begin{tabular}{lcccc}
\hline & Verão & Outono & Inverno & Primavera \\
\hline Verão & 0 & 0,5609 & 0,3698 & 0,1392 \\
Outono & $0,0347^{*}$ & 0 & $0,0241^{*}$ & $0,022^{*}$ \\
Inverno & 0,0822 & 0,2673 & 0 & 0,257 \\
Primavera & 0,7433 & $0,0157^{*}$ & 0,1142 & 0 \\
\hline
\end{tabular}

$(*)$ Valores com diferença estatística.

O índice de diversidade de Shannon (Tabela 5) foi maior para o inverno $\left(H^{\prime}=1,68\right)$, o que pode ser explicado pelo menor valor de dominância encontrado $(\mathrm{D}=0,31)$. Em relação aos estimadores de riqueza, no verão foram amostrados 63-95\%; no outono, 88-97\%; no inverno, 90-93\%, e na primavera, 79-93\%. Analisando os dados climáticos (Tabela 3), observamos que as temperaturas variam, de acordo com as estações, sendo a mais baixa no inverno $\left(7,4^{\circ} \mathrm{C}\right)$ e a mais alta no verão $\left(26^{\circ} \mathrm{C}\right)$.

TABELA 5: Riqueza, abundância, índices de diversidade, dominância e estimadores de riqueza (Chao 2, Jackknife 1 e Jackknife 2) dos coleópteros amostrados com armadilha de queda, entre janeiro de 2011 e janeiro de 2012, em fragmento de restinga, no extremo sul do Rio Grande do Sul, Brasil.

\begin{tabular}{|c|c|c|c|c|}
\hline & Verão & Outons & Inverno & rimavera \\
\hline Riqueza Média & 52 & 30 & 25 & 52 \\
\hline $\mathbf{N}$ & 2113 & 718 & 664 & 3459 \\
\hline $\begin{array}{l}\text { Diversidade de } \\
\text { Shannon-Wiener (H') }\end{array}$ & 1,05 & 1,53 & 1,68 & 1,29 \\
\hline $\begin{array}{l}\text { Dominância de } \\
\text { Simpson (D) }\end{array}$ & 0,60 & 0,36 & 0,31 & 0,49 \\
\hline Chao 2 & 62,30 & 33,38 & 27,31 & 66,75 \\
\hline Jackknife 1 & 55,70 & 31,88 & 26,98 & 56,25 \\
\hline Jackknife 2 & 83,08 & 34,54 & 28,24 & 59,23 \\
\hline
\end{tabular}

\section{Discussão}

$\mathrm{Na}$ área de estudo, somente há estudos sobre a fauna de Lepidoptera (SILVA et al., 2012) e Aranae (RODRIGUES, 2005). Portanto, todas as espécies de coleópteros encontradas neste trabalho são registros iniciais para a literatura. A família mais abundante foi Nitidulidae que, segundo Arnett Jr et al. (2002), possui aproximadamente 2.800 espécies em 172 gêneros no mundo, das quais pouco mais de 700 são da região Neotropical (LIMA, 1953). Teixeira et al. (2009) amostraram essa família como mais abundante e em número bem semelhante a este estudo (4.782 indivíduos), em Mata Atlântica, no Rio de Janeiro, no período de 1 ano. A ocorrência de poucas famílias muito abundantes, incluindo Nitidulidae, deve ser atribuída a uma melhor adaptação em ambientes fragmentados de florestas ou áreas degradadas (MEDRI; LOPES, 2001). A área de estudo sofreu grande influência antrópica ao longo de sua história, inclusive passando por um projeto paisagístico (LUIS; BERTELS, 1951). Segundo Athié e Paula (2002), a maior parte das espécies de Nitidulidae são decompositores de matéria orgânica, a grande abundância dessa família durante o período de coleta talvez seja decorrente de grande oferta de material em decomposição no ambiente estudado. Ganho e Marinoni (2003) atribuem que o fato de espécies pertencentes a uma determinada família serem mais abundantes do que outras pode significar que fatores como disponibilidade trófica do ambiente estão tendendo a favorecê-las. Durante um ano de amostra foram verificadas duas situações sazonais em que a primavera teve o mesmo número de morfoespécies que o verão e maior número de indivíduos amostrados; esse resultado é semelhante ao encontrado por Dummel et al. (2011), que capturaram maior número de indivíduos de setembro a janeiro em áreas de restinga, na cidade de Rio Grande, RS. $\mathrm{Na}$ análise de similaridade geral, os valores do teste foram muito próximos de zero, evidenciando que a composição de espécies e a abundância são similares entre as estações.

Apesar do pequeno número de indivíduos amostrados neste trabalho, o índice de diversidade de Shannon foi maior para o inverno, devido à sensibilidade ao alto número de espécies raras. As 25 
famílias amostradas neste estudo possuem hábitos variados como decompositores (Nitidulidae), predadores (Carabidae), detritívoros (Scarabaeidae) e herbívoros (Chrysomelidae), características dos besouros, indicando que o ambiente da área de estudo possui oferta alimentar variada para abrigar essa fauna. Aqui verificamos que a comunidade de coleópteros pode ser afetada pelas baixas temperaturas, nas estações mais frias, o que já era esperado. Porém, mesmo com a baixa abundância, ainda ocorreu um número considerável de espécies. A observação da dinâmica de áreas florestais exige que novos estudos sejam realizados a fim de se obter mais dados sobre a biodiversidade da região. A fauna de coleópteros foi representada por 25 famílias e a comunidade de solo parece ser influenciada pelas variações de temperatura médias que diferem ao longo do ano.

\section{Referências}

ARNETT JR, R. H.; THOMAS, M. C.; SKELLEY, P. E.; FRANK, J. H. American beetles. Vol. 2. Polyphaga: Scarabaeoidea through Curculionoidea. Boca Raton: CRC, 2002. 839 p.

ATHIÉ, I.; DE PAULA, D. C. Insetos de grãos armazenados: aspectos biológicos e identificação. São Paulo: Varela, 2002. 244 p. CASARI, A. S.; IDE, S. Coleoptera. In. RAFAEL, J. A; MELO, G. A. R.; CARVALHO, C. J. B; CASARI, S. A.; CONSTANTINO, R. (Ed.). Insetos do Brasil: diversidade e taxonomia. Ribeirão Preto: Holos Editora, 2012. p. 470-552.

CLARKE, K. R. Non-parametric multivariate analyses of changes in community structure. Australian Journal of Ecology, Carlton, v. 18, n. 1, p. 117-143, 1993.

DUMMEL, K.; OLIVEIRA, E. A.; ZARDO, C. M. L. Variação de abundância, diversidade ecológica e similaridade de Coleoptera (Insecta) entre restinga e marisma do Estuário da Lagoa dos Patos, Rio Grande, RS. EntomoBrasilis, Viçosa, v. 4, n. 2, p. 39-44, 2011.

EMBRAPA - LABORATÓRIO DE AGROMETEOROLOGIA. Estação Agroclimatológica de Pelotas. Disponível em $<$ http:// www.cpact.embrapa.br/agromet/estacao/mensal.html $>$. Acesso em: 15 jan. 2016.

GANHO, N. G.; MARINONI, R. C. Fauna de Coleoptera no Parque Estadual de Vila Velha, Ponta Grossa, Paraná, Brasil. Abundância e riqueza das famílias capturadas através de armadilhas malaise. Revista Brasileira de Zoologia, Curitiba, v. 20, n. 4, p. 727-736, 2003.

HAMMER, Ø.; HARPER, D. A. T.; RYAN, P. D. PAST: Paleontological Statistics Software Package for education and data analysis. Paleontologia Electronica, Oslo, v. 4, n 1, p. 1-9, 2001.

KEVAN, P. G.; BAKER, H. G. Insects as flower visitors and pollinators. Annual Review of Entomology, Palo Alto, v. 28, n. 1, p. $407-453,1983$.
LEWINSOHN, T. M.; PRADO, P. I. How many species are there in Brazil? Conservation Biology, San Francisco, v. 19, n. 3, p. 619$624,2005$.

LIMA, A. D. C. Insetos do Brasil. 8ํㅜ Tomo. Coleópteros, 2a Parte. Rio de Janeiro: Escola Nacional de Agronomia, 1953. 323 p.

LUIS, T.; BERTELS, A. Horto Botânico do Instituto Agronômico do Sul (Pelotas) - Guia dos visitantes. Pelotas: Instituto Agronômico do Sul, 1951.93 p.

MEDRI, Í. M.; LOPES, J. Scarabaeidae (Coleoptera) do Parque Estadual Mata dos Godoy e de área de pastagem, no norte do Paraná, Brasil. Revista Brasileira de Zoologia, Curitiba v. 18, p. 135-141, 2001.

NOGUEIRA, E. M. L.; DE ARRUDA, V. L. V. Fenologia reprodutiva, polinização e sistema reprodutivo de Sophora tomentosa L. (Leguminosae - Papilionoideae) em restinga da praia da Joaquina, Florianópolis, sul do Brasil. Biotemas, Florianópolis, v. 19, n. 2, p. 29-36, 2006.

PEARSON, D. L. Selecting indicator taxa for the quantitative assessment of biodiversity. Philosophical Transactions of the Royal Society of London B: Biological Sciences, London, v. 345, n. 1311, p. 75-79, 1994.

PEEL, M. C.; FINLAYSON, B. L.; MCMAHON, T. A. Updated world map of the Köppen-Geiger climate classification. Hydrology and Earth System Sciences, Göttingen, v. 4, n. 2, p. 439-473, 2007.

RODRIGUES, E. N. L. Araneofauna de serapilheira de duas áreas de uma mata de restinga no município de Capão do Leão, Rio Grande do Sul, Brasil. Biotemas, Florianópolis, v. 18, n. 1, p. 73 92, 2005.

SCHERER, K. Z.; ROMANOWSKI, H. P. Predação de Megacerus baeri (Pic, 1934) (Coleoptera: Bruchidae) sobre sementes de Ipomoea imperati (Convolvulaceae), na praia da Joaquina, Florianópolis, sul do Brasil. Biotemas, Florianópolis v. 18, n. 1, p. 39-55, 2005.

SILVA, J. M.; DA CUNHA, S. K.; ELY, E. J.; GARCIA, F. R. M. Borboletas frugívoras (Lepidoptera: Nymphalidae) no Horto Botânico Irmão Teodoro Luis, Capão do Leão, Rio Grande do Sul, Brasil. Biotemas, Florianópolis, v. 26, n. 1, p. 87-95, 2012.

SLIPINSKI, A.; LESCHEN, R. A. B.; LAWRENCE, J. F. Order Coleoptera Linnaeus, 1758. In: ZHANG, Z.-Q. (Ed.). Animal biodiversity: an outline of higher-level classification and survey of taxonomic richness. Zootaxa, Auckland, 3148, 2011. p. 203-208.

SPEIGHT, M. R.; HUNTER, M. D.; WATT, A. D. Ecology of insects: concepts and applications. Oxford: Blackwell Science, 1999. $350 \mathrm{p}$.

TEIXEIRA, C. C. L.; HOFFMANN, M.; SILVA-FILHO, G. Comunidade de Coleoptera de solo em remanescente de Mata Atlântica no estado do Rio de Janeiro, Brasil. Biota Neotropica, Campinas, v. 9, n. 4, p. 91-95, 2009.

TRIPLEHORN, C. A.; JOHNSON, N. F. Estudo dos insetos. São Paulo: Cengage Learning, 2011. 810 p.

VEloso, H. P.; RANGEL FILHO, A. L. R.; LIMA, J. C. A. Classificação da vegetação brasileira, adaptada a um sistema universal. Rio de Janeiro: IBGE, Departamento de Recursos Naturais e Estudos Ambientais, 1991. 124 p. 